\title{
Fluid Mobility over Corrugated Surfaces in the Stokes Regime
}

\author{
S. Shelley, ${ }^{1}$ J. D. Smith, ${ }^{2}$ A. P. Hibbins, ${ }^{1}$ J. R. Sambles,${ }^{1}$ and S. A. R. Horsley ${ }^{1}$ \\ 1) Department of Physics and Astronomy, University of Exeter, Stocker Road, \\ Devon, Ex4 4QL, United Kingdom \\ 2) DSTL, Porton Down, Salisbury, Wiltshire, SP4 OJQ, \\ United Kingdom
}

(Dated: 29 April 2016)

An exact solution is found for laminar fluid flow along the grooves of a family of surfaces whose shape is given by the Lambert W-function. This simple solution allows for the slip length in the direction parallel to the grooves to be calculated exactly. With this analytical model we establish the regime of validity for a previously untested perturbation theory intended for calculating the surface mobility tensor of arbitrary periodic surfaces, finding that it compares well to the exact expression for nearly all choices of parameters of the conformal map. To test this perturbation theory further, the mobility tensor is evaluated for a simple sinusoidal surface for flow both parallel and perpendicular to the grooves, finding that the perturbation theory is less accurate in the latter of these two cases. 


\section{INTRODUCTION}

In fluid mechanics, the standard boundary condition on the fluid velocity is the so called no-slip condition where, over a flat solid body there is zero relative velocity between the fluid and the surface. Yet in some situations it is more appropriate to assume interfacial slip, where the fluid has a non-zero relative velocity along the surface. Being able to control interfacial slip is useful for example, in reducing the drag around objects ${ }^{1}$. Conventionally the slip velocity $\left(\mathbf{u}_{s}\right)$, i.e. the velocity the fluid moves along the surface, is characterised by a so called 'slip length' $b$, which is the required linear extrapolation into the solid, necessary for the velocity to vanish. It is defined according to the equation

$$
\mathbf{u}_{s}=b \frac{\partial \mathbf{u}}{\partial n}
$$

where $\partial \mathbf{u} / \partial n$ is the fluid's velocity gradient in the direction normal to the surface, $\hat{\mathbf{n}}$. A tensorial form of (1) is generally needed however to allow the slip properties of a surface to become anisotropic, leading to the concept of the surface mobility tensor ${ }^{2}$. The surface mobility tensor includes information regarding the slip length in each direction. The relation between the surface mobility tensor $(\underline{\underline{M}})$ and the slip velocity is given by

$$
\mathbf{u}_{s}=-\mu^{-1} \underline{\underline{M \tau}} \boldsymbol{\underline { \tau }}
$$

where $\underline{\underline{M}}$ is a $2 \times 2$ symmetric tensor with the dimensions of length that acts in the plane of the surface, $\boldsymbol{\tau}$ is the in-plane shear stress within the fluid, and $\mu$ is the dynamic viscosity. Re-

cent work in this context has primarily dealt with superhydrophobic surfaces ${ }^{3-6}$, that utilise an air layer trapped in nanoscale roughness to produce slip. A key issue with such superhydrophobic surfaces is their ability to retain a trapped gas layer and thus maintain the slip.

Another method of increasing the slip is by structuring the surface. In nature, shark skin is known for its drag reducing properties and idealised models of shark scales ${ }^{7}$ or sinusoidal riblets $^{8}$, which mimic the top of the scales, have been developed to give drag reduction and delay boundary layer separation. However, a theoretical understanding of the observed flow is generally lacking due to the complexity of both the surface structures and solving the Navier-Stokes in the turbulent regime. The regime of low Reynolds numbers (Stokes flow) is analytically much more tractable and is the focus of this paper. 
Analytically, a problem limited to Stokes' flow is the simplest case for exploring how structured surfaces influence flow, however it is important to remember that in this regime any such structuring will only increase the drag on the surface ${ }^{9}$. This problem has been approached by many authors: Wang examined Stokes flow through channels bounded by one- and two-dimensional sinusoidally structured surfaces ${ }^{10,11}$ and developed equations for the flow in each direction using perturbation analysis. Using the techniques of complex analysis, Scholle ${ }^{12}$ found an exact solution for the flow through a one-dimensionally structured sinusoidal channel of arbitrary channel height and "waviness". Both were able to use their analysis to find exact expressions for the slip length of the surface. Niavarani et al. ${ }^{13}$ modelled flow through a sinusoidal channel of varying amplitude and intrinsic slip to explore the effect on vortex formation above the surface. For more general problems Kamrin et al. ${ }^{14}$ developed a perturbation theory to find a general equation for the mobility tensor $(\underline{\underline{\underline{M}}})$ in terms of the surface shape. This is not generally valid, providing an approximation only up to an aspect ratio defined by the surface profile.

In the present work, we analyse the flow along the grooves of a family of one-dimensionally structured surfaces, and find the exact slip length for these surfaces. We then compare this result with the predictions from both a numerical finite element method model and the perturbation theory developed by Kamrin et al. We find convincing agreement between the analytic results, the perturbation theory and the numerical simulations at low aspect ratios. We then explore flow perpendicular to a sinusoidally varying surface, demonstrating the breakdown of the perturbation theory, and explore this failure as further terms are included in the perturbation theory.

\section{CALCULATING SLIP LENGTH FROM A CONFORMAL MAP}

\section{A. Flow}

In the Stokes regime, where viscous forces dominate, the flow of fluid is described by the Stokes equation,

$$
\nabla^{2} \mathbf{u}=\frac{1}{\mu} \nabla \mathrm{p}
$$


where $\mathbf{u}=(u, v, w)$ and $\mathrm{p}$ are the fluid velocity and pressure respectively and $\mu$ is the fluid's dynamic viscosity. If a system is translationally invariant along the $\hat{\mathbf{z}}$ axis, and the flow is driven by a moving surface at $y=H$ then the pressure will be $z$ independent and (3) can be reduced to Laplace's equation for flow along $\hat{\mathbf{z}}$

$$
\nabla^{2} w=0
$$

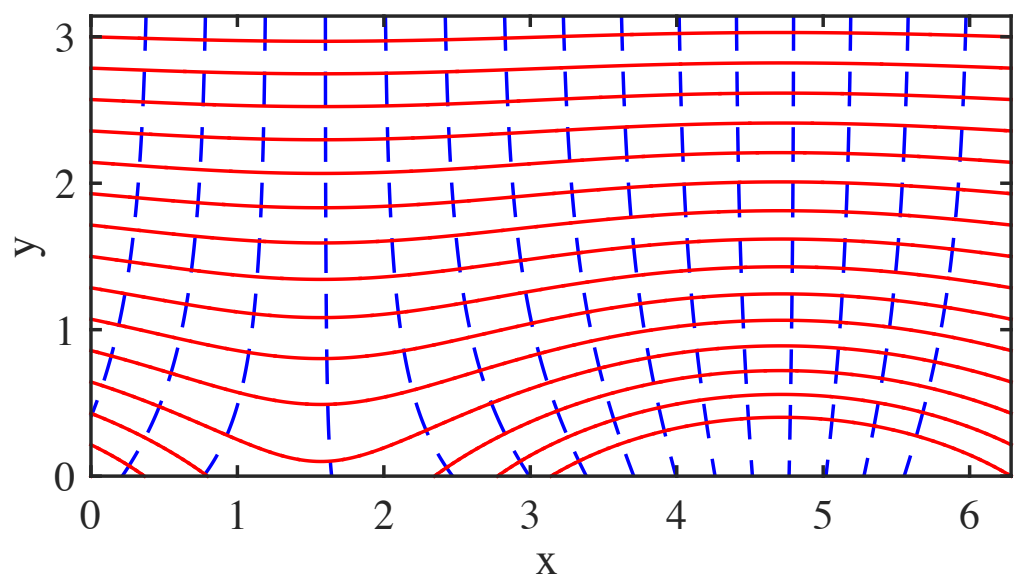

FIG. 1. Lines of constant $\operatorname{Re}[\tilde{z}]$ (dashed blue) and $\operatorname{Im}[\tilde{z}]$ (solid red) in the $\mathrm{x}-\mathrm{y}$ plane for $\alpha=0.6$. Flow in or out of the page over a surface following the contour of any of the solid lines can be mapped to the solution of flow over a flat plate lying in the x-z plane.

By representing the $x-y$ coordinate system as a single complex number $z=x+\mathrm{i} y$, Laplace's equation can be written as $\partial^{2} w / \partial z \partial z^{\star}=0$, the solution of which is any function of solely $z$ or $z^{\star}$. It also means that we can perform a conformal coordinate map, $z \rightarrow \tilde{z}(z)$, and leave the form of Laplace's equation unchanged ${ }^{15}$. Thus the solution for the flow over a flat plate can be mapped to the new coordinate system to give the flow along the grooves of a structured surface. To develop an analytic formula for the slip length over a corrugated surface we start with a simple periodic conformal map

$$
\tilde{z}=z+\alpha \mathrm{e}^{\mathrm{i} k z},
$$


where $\alpha$ is a constant, $k=2 \pi / L$ and $L$ is the periodicity of the surface (note: this complex number $z$ that represents the $x-y$ plane should not be confused with the direction of flow which is in the $\hat{z}$ direction). The lines of constant $\operatorname{Re}[\tilde{z}]$ and $\operatorname{Im}[\tilde{z}]$ in the $x-y$ plane are then given by the following equations:

$$
\begin{aligned}
& \operatorname{Re}[\tilde{z}]=\tilde{x}_{0}=x+\alpha \cos (k x) \mathrm{e}^{-k y}, \\
& \operatorname{Im}[\tilde{z}]=\tilde{y}_{0}=y+\alpha \sin (k x) \mathrm{e}^{-k y},
\end{aligned}
$$

which are shown in FIG. 1. The surfaces defined by (7) are approximately flat for large positive values of $\tilde{y}_{0}$ and become more curved as $\tilde{y}_{0}$ decreases, eventually ceasing to be continuous lines and no longer giving a useful representation of a surface.

For flow driven by a plate moving at velocity $\mathrm{V}$, only in the $\hat{z}$ direction located at a height $H$ above a stationary flat plate located at height $y_{0}$ with respect to $y=0$ (Couette flow), the $z$ component of the velocity at height $\mathrm{y}$ is

$$
w=\frac{V}{H}\left(y-y_{0}\right),
$$

Now consider flow over the structured surface $\tilde{y}_{0}=y+\alpha \sin (k x) \mathrm{e}^{-k y}$. It is clear that at a height far from the bottom surface the transformed coordinate lines become flat in the conformal map (FIG. 1) and hence we can continue to assume a a flat driving plate and Couette flow. Using the conformal map, the solution above a surface located at $\tilde{y}_{0}$, defined by (7), is given by simply translating the equation for Couette flow, (8), into the new coordinate system,

$$
w=\frac{V}{H}\left(y+\alpha \sin (k x) \mathrm{e}^{-k y}-\tilde{y}_{0}\right) .
$$

The flow goes to zero along the structured surface and is equal to $V$ along $y=\tilde{y}_{0}+H$. At the top surface the exponential can be neglected and the flow takes the form as if the lower surface is flat and located at $\tilde{y}_{0}$. This result can be readily extended to more complicated maps written as an arbitrary sum of complex exponentials.

\section{B. Slip Length}

From the compact expression for the flow (9) it is possible to develop an analytic expression for the slip length $b_{z}$ as defined in (1). In this paper we adopt the convention that a 


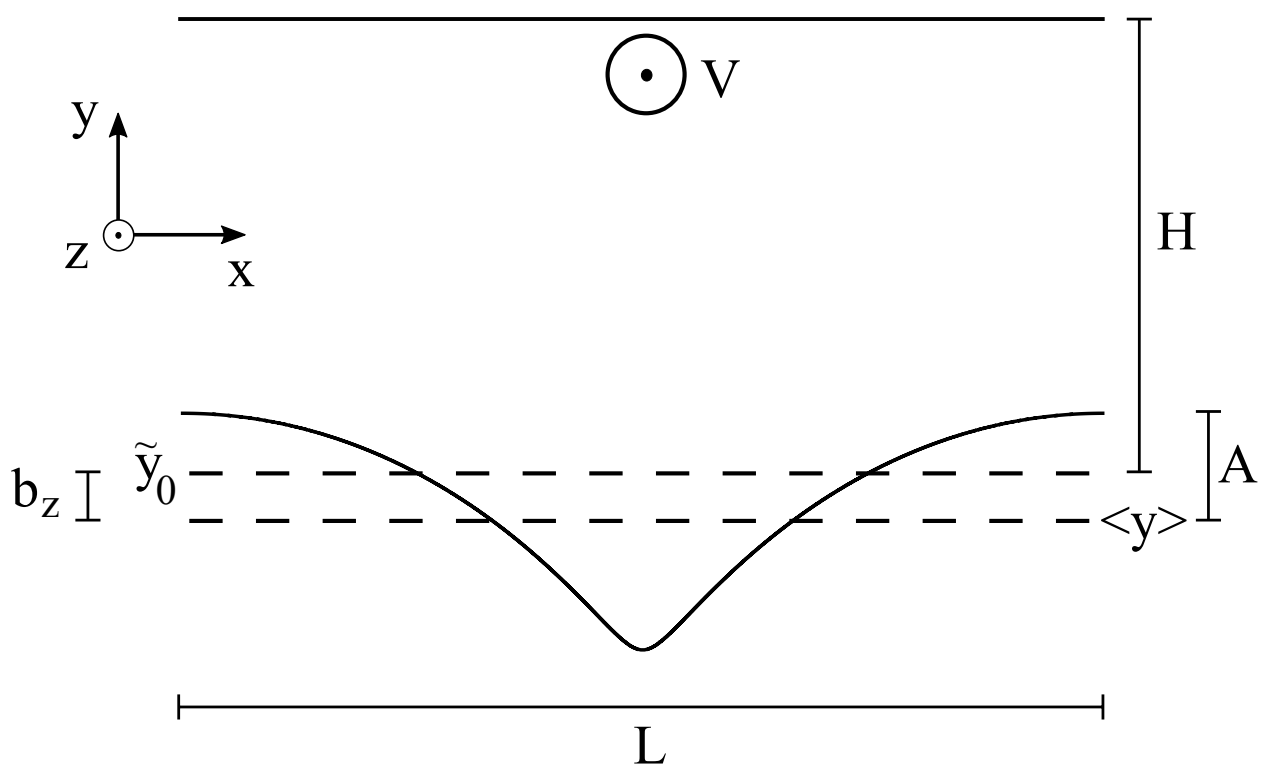

FIG. 2. Flow in the $\hat{z}$ direction over a one-dimensionally structured surface given by the lines of constant $\tilde{y}_{0}=y+\alpha \sin (k x) \mathrm{e}^{-k y}$, is driven by a flat plate moving at constant speed $V$ located a distance $H$ above it. The surface shown is for $\tilde{y}_{0}=0.5, \alpha=0.6$ and $L=2 \pi$ The slip length, $b_{z}$, is the difference between the true average surface height $\langle y\rangle$ and the apparent one $\tilde{y}_{0}$. The amplitude $A$ of the surface is given by the coefficient of the first order term in the Fourier series representation of the surface.

subscript on $b$ denotes a slip length in a particular direction. The equation for the bottom surface over which the fluid flows (7) can be rewritten as,

$$
\left[y(x)-\tilde{y}_{0}\right] \mathrm{e}^{k y(x)}=-\alpha \sin (k x) .
$$

which is the form of the well-studied functional relationship $\mathrm{W} \exp (\mathrm{W})=x$, which has a solution in terms of the Lambert $\mathrm{W}$-function ${ }^{16}$,

$$
y(x)=\tilde{y}_{0}+\frac{1}{k} W\left(-\alpha k \sin (k x) \mathrm{e}^{-k \tilde{y}_{0}}\right) .
$$

The function $W$ takes complex values when its argument becomes less than $1 / e\left(\operatorname{see}^{16}\right)$, which is when the lower surface shown in FIG. 2 ceases to be continuous. The true average surface height is then

$$
\langle y\rangle=\tilde{y}_{0}+\frac{1}{2 \pi} \int_{0}^{L} W\left(-\alpha k \sin (k x) \mathrm{e}^{-k \tilde{y}_{0}}\right) d x .
$$

The slip length is then the difference between the true average surface height $\langle y\rangle$ and the apparent one $\tilde{y}_{0}$ where an observer at the top surface would expect the flow to go to zero 


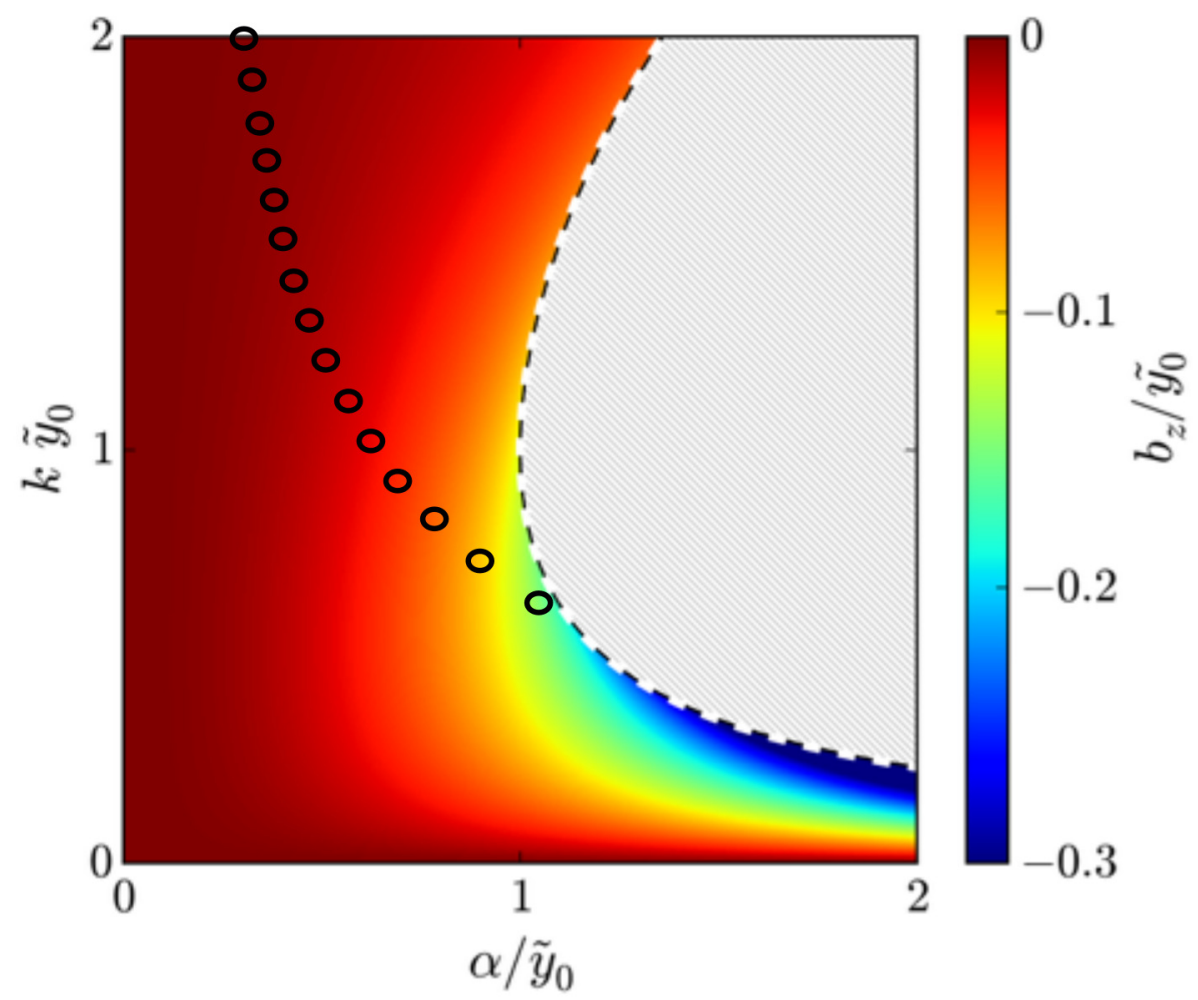

FIG. 3. Slip length $b_{z}$ as a function of $k$ and $\alpha$, numerically computed from $(13)^{17}$. The hatched region indicates the range of parameters where the conformal map ceases to represent a continuous surface (the boundary of this region is where $\alpha=\mathrm{e}^{k \tilde{y}_{0}-1} / k$, which is when the Lambert function ceases to return real values). Circles show the position of the conformal surfaces analysed in the FEM modelling later in the paper. This line was chosen as it allows us to explore the full range of aspect ratios avaliable to the conformal map. All variables are normalized in terms of the apparent surface position $\tilde{y}_{0}$.

for a flat bottom plate,

$$
b_{z}=\langle y\rangle-\tilde{y}_{0}=\frac{1}{2 \pi} \int_{0}^{L} W\left(-\alpha k \sin (k x) \mathrm{e}^{-k \tilde{y}_{0}}\right) d x .
$$

Using the series expansion of $W(x)^{16}$,

$$
W(x)=\sum_{n=1}^{\infty}(-1)^{n-1} \frac{n^{n-2}}{(n-1) !} x^{n},
$$

an exact equation for the slip length is obtained

$$
b_{z}=-\sum_{n=1}^{\infty} \frac{(2 n)^{2 n-2}}{(2 n-1) !} \frac{\left(\alpha k \exp \left(-k \tilde{y}_{0}\right)\right)^{2 n}}{k} \frac{(2 n-1) ! !}{(2 n) ! !}
$$


where the odd $n$ terms in the series (14) integrate to zero and the two exclamation marks represent the double factorial. This form of the slip length exponentially decays with increasing $\tilde{y}_{0}$ because, as can be seen from (7) this limit corresponds to an exponential flattening of the surface. From this analysis it is clear that the slip length $b_{z}$ is always negative (all the terms in the series (15) are positive, and this series is multiplied by minus one). This means that far above the surface the flow is unaffected by the structuring except that the velocity $w$ is consistent with the bottom surface being at a distance $H$, which is always closer than the true value of $H+\tilde{y}_{0}-\langle y\rangle$, thus increasing the drag above that of a flat surface. FIG. 3 shows how the slip length (13) varies as a function of $\alpha$ and $k$, illustrating that the largest magnitude of slip length (relative to the apparent position $\tilde{y}_{0}$ ) is achieved when $k \tilde{y}_{0} \ll 1$ and $\alpha / \tilde{y}_{0} \gg 1$.

As this exact expression is an infinite series it is worth looking at where it converges. Taking the ratio of the $n$th and $(n+1)$ th term we get,

$$
\frac{b_{n+1}}{b_{n}}=\left(1+\frac{1}{n}\right)^{2 n-1}\left[\alpha k e^{-k \tilde{y}_{0}}\right]^{2} \text {. }
$$

As $n \rightarrow \infty$ this reduces to

$$
\left[\alpha k e^{-k \tilde{y}_{0}}\right]^{2}
$$

which is less than 1 (i.e. converges) as long as $\exp \left(-k \tilde{y}_{0}\right)<\frac{1}{\alpha k}$. We find that for all surfaces considered in this paper, excellent convergence can be achieved with the inclusion of just 10 terms in the series. However all results presented in this paper using the exact solution use 72 terms as an arbitrary limit.

It is straightforward to see that any surface derived from a conformal map will have a negative slip length: the conformal transformation must tend to the identity as $y \rightarrow \infty$ and be analytic above the curve which defines the shape of the surface. Therefore if the surface has periodicity $L$, then above the surface we are restricted to maps of the form $\tilde{z}(z)=z+\sum_{n} z_{n} \exp (\mathrm{i} k n z)$, where $z_{n}$ are arbitrary complex constants. The lines of constant $\tilde{y}: \tilde{y}_{0}=y+\sum_{n} \operatorname{Im}\left[z_{n} \exp (\mathrm{i} k n x)\right] \exp (-k n y)$ define the surface shapes, the flow over which appears, to an observer fixed at the upper surface, to be as over a flat surface positioned at $\tilde{y}_{0}$. The values of $y$ generally tend to lie more frequently below $\tilde{y}_{0}$ rather than above, 
implying a negative slip length. To see this consider the inverse transformation,

$$
z(\tilde{z})=\tilde{z}+\sum_{n=1}^{\infty} \tilde{z}_{n} \mathrm{e}^{\mathrm{i} k n \tilde{z}}
$$

using this expression the average surface height $\langle y\rangle$ for a fixed $\tilde{y}=\tilde{y}_{0}$ can be computed exactly

$$
\begin{aligned}
\langle y\rangle & =\tilde{y}_{0}+\frac{1}{L} \int_{0}^{L} \sum_{n=1}^{\infty} \operatorname{Im}\left[\tilde{z}_{n} \mathrm{e}^{\mathrm{i} k n \tilde{x}}\right] \mathrm{e}^{-k n \tilde{y}} \frac{d x}{d \tilde{x}} d \tilde{x} \\
& =\tilde{y}_{0}-\frac{1}{L} \sum_{n=1}^{\infty} k n \mathrm{e}^{-2 k n \tilde{y}_{0}} \int_{0}^{L} \operatorname{Im}\left[\tilde{z}_{n} \mathrm{e}^{\mathrm{i} k n \tilde{x}}\right]^{2} d \tilde{x}
\end{aligned}
$$

and is clearly always less than the apparent surface height $\tilde{y}_{0}$.

(In passing we note an interesting equivalence between this slip length calculation and the electrostatics of parallel corrugated plates, where the expression for the flow velocity $w$ would translate into an electrostatic potential. If we have a parallel plate capacitor, where the two plates are seperated by a distance $H$, and then corrugate the lower plate we would need to increase the average seperation so as to maintain the same electric field at the top plate.)

\section{NUMERICAL ANALYSIS AND COMPARISON WITH PERTURBATION THEORY}

In this section we compare our simple analytic treatment of the slip length with the results of numerical modelling and a recently developed perturbation theory, with the aim of gaining some insight into the regime of validity of the perturbation theory. Using an FEM numerical model ${ }^{18}$, the value of the predicted slip length for the surfaces discussed in the previous section were compared against expression (15). Couette flow parallel to the average plane of the structured surface was induced at a fixed height of $40-\tilde{y}_{0} \mathrm{~mm}$ at a speed of $5 \times 10^{-5} \mathrm{~ms}^{-1}$. The values for $\alpha$ and $L$ were taken as $0.5 \mathrm{~mm}$ and $2 \pi \mathrm{mm}$ respectively and then the values of velocity $(w)$ and shear stress $\left(\tau_{z}\right)$ in the $\hat{z}$ direction were extracted. To calculate the slip length $b_{z}$ of the surface in the FEM model, the following equation was 
used:

$$
b_{z}=\frac{\mu w}{\tau_{z}}-\left(H_{0}-\langle y\rangle\right)
$$

where $H_{0}$ is the position of the top surface. Remembering that the shear stress is the velocity gradient times the dynamic viscosity, it is clear that the above definition of the slip length (19) is equivalent to the earlier expression (13). This equation allows one to extract the slip length by using the linear velocity gradient at the top surface of the model.

We also compare the analytic results of the previous section with the perturbation theory developed by Kamrin et al. ${ }^{14}$ who consider flow over an arbitrary periodic surface and find the following approximation to the mobility tensor,

$$
\underline{\underline{\underline{M}}}=\left(\begin{array}{cc}
\tilde{M}_{11} & \tilde{M}_{12} \\
\tilde{M}_{21} & \tilde{M}_{22}
\end{array}\right),
$$

where,

$$
\begin{aligned}
\tilde{M}_{11} & =\sum_{(m, n) \neq 0} \frac{2 k_{m}^{2}+k_{n}^{2}}{\sqrt{k_{m}^{2}+k_{n}^{2}}}|\hat{h}(m, n)|^{2}, \\
\tilde{M}_{12}=\tilde{M}_{21} & =\sum_{(m, n) \neq 0} \frac{k_{m} k_{n}}{\sqrt{k_{m}^{2}+k_{n}^{2}}}|\hat{h}(m, n)|^{2}, \\
\tilde{M}_{22} & =\sum_{(m, n) \neq 0} \frac{k_{m}^{2}+2 k_{n}^{2}}{\sqrt{k_{m}^{2}+k_{n}^{2}}}|\hat{h}(m, n)|^{2},
\end{aligned}
$$

and where it has been assumed that the aspect ratio of the surface is always small. Here $k_{m}=2 \pi m / L_{x}$ and $k_{n}=2 \pi n / L_{z}$, with $L_{x}$ and $L_{z}$ being the periodicity of the surface in $x$ and $z$ respectively, and $\hat{h}(m, n)$ are the Fourier components of the surface height $h(x, z)$. In this work we only consider surfaces that are periodic in one direction (i.e. $L_{z}=\infty, k_{n}=0$ )so this reduces down to

$$
\underline{\underline{\underline{M}}}=\left(\begin{array}{cc}
\tilde{M}_{11} & 0 \\
0 & \tilde{M}_{22}
\end{array}\right)
$$

where,

$$
\tilde{M}_{11}=2 \tilde{M}_{22}=\sum_{m \neq 0} k_{m}|\hat{h}(m)|^{2} .
$$

From this point on we shall just refer to terms in the mobility tensor but note that $M_{11}$ and $M_{22}$ are equivalent to $b_{x}$ and $b_{z}$ respectively. 
Using the definition of the surface shape (11) the Fourier representation of the Lambert W function is found to be

$$
\begin{aligned}
y(x) & =\sum_{m=-\infty}^{\infty} \hat{h}(m) \mathrm{e}^{\mathrm{i} k m x}=\tilde{y}_{0}+\frac{1}{k} W\left(-\alpha k \sin (k x) \mathrm{e}^{-k \tilde{y}_{0}}\right) \\
\rightarrow \hat{h}(m) & =\tilde{y}_{0} \delta_{m 0}-\frac{1}{k} \sum_{p=0}^{\infty}(-1)^{p} \frac{(m+2 p)^{m+2 p-2}}{(m+2 p-1) !}\left(\frac{\alpha k \mathrm{e}^{-k \tilde{y}_{0}}}{2 \mathrm{i}}\right)^{m+2 p}\left(\begin{array}{c}
m+2 p \\
p
\end{array}\right)
\end{aligned}
$$

to which we can apply equation (24) to calculate the mobility tensor and thus the slip length. FIG. 4 demonstrates that there is good agreement between the three solutions for the slip length, but that for larger aspect ratios the predictions of expression (24) diverge slightly from the FEM model and exact solution: a maximum difference of $11 \%$ is found for the largest aspect ratio tested of 0.056. In this figure the amplitude of the surface $(A)$ was taken as the coefficient of the first order term in the Fourier series representation of the surface. Note that FIG. 4 covers the full range of surfaces that can be reached with the conformal map (5), and the perturbative method of calculating the slip is very accurate for this entire range, beginning to fail only for the largest aspect ratios when sharp features also begin to develop in the surface profile.

As the available aspect ratios for this family of Lambert $W$ surfaces is limited, it is of interest to explore the application of the perturbation theory to more general surface profiles where there is no analytic solution. We examine the case of the slip length for a sinusoidal surface

$$
y(x)=A \sin (k x)
$$

where in this case the large aspect ratios are not associated with sharp features in the surface, as they were for the surfaces we generated from the conformal map. With this simple surface we are able to use the FEM model to explore the limit at which the perturbation analysis (22) fails to accurately predict the slip length. Equation (19) is again used to calculate the slip length from the FEM model in the $\hat{\boldsymbol{x}}$ and $\hat{\boldsymbol{z}}$ directions. Based on expression (24) the mobility tensor for the sinusoidal surface reduces to

$$
\underline{\underline{M}}=\frac{A^{2} \pi}{L^{2}}\left(\begin{array}{ll}
2 & 0 \\
0 & 1
\end{array}\right) .
$$




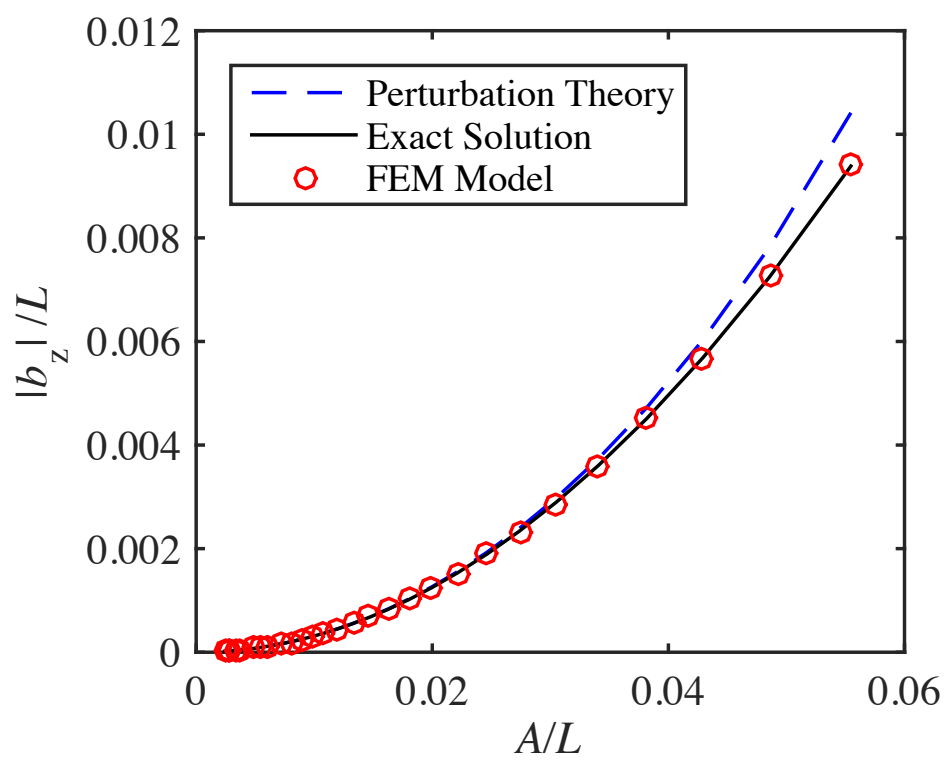

FIG. 4. Comparison between the modulus of the exact values for the slip length and values from the FEM model for surfaces defined by $\tilde{y}_{0}=y+\alpha \sin (k x) \mathrm{e}^{-k y}$. Values derived using expression (24) from the perturbation theory are also shown. At low aspect ratios $(A / L)$ these agree very well with the exact solution and the FEM model, however as the aspect ratio increases the values start to diverge, overestimating the slip length. A maximum difference of $11 \%$ is found for the largest aspect ratio tested of 0.056

For the sinusoidal surface we shall look at the flow both across and along the grooves of the surface so as to explore each term in the mobility tensor separately to give an overall picture of its validity.

First focusing on $M_{22}$, corresponding to flow along the grooves of the structure, observe that at low aspect ratios there is again excellent agreement between the FEM predictions and the predictions of expression (24) (FIG. 5a). However we now see that as the aspect ratio increases, the perturbation theory significantly diverges from the numerical results: using the arbitrary difference of $10 \%$ to characterise a significant divergence between the solutions, the perturbation expansion analysis is accurate up to aspect ratios $(A / L)$ of 0.1 .

It is also worth exploring $M_{11}$ for the sinusoidal surface where the fluid flow is across the corrugations. In this case the perturbation theory is much less effective: diverging 

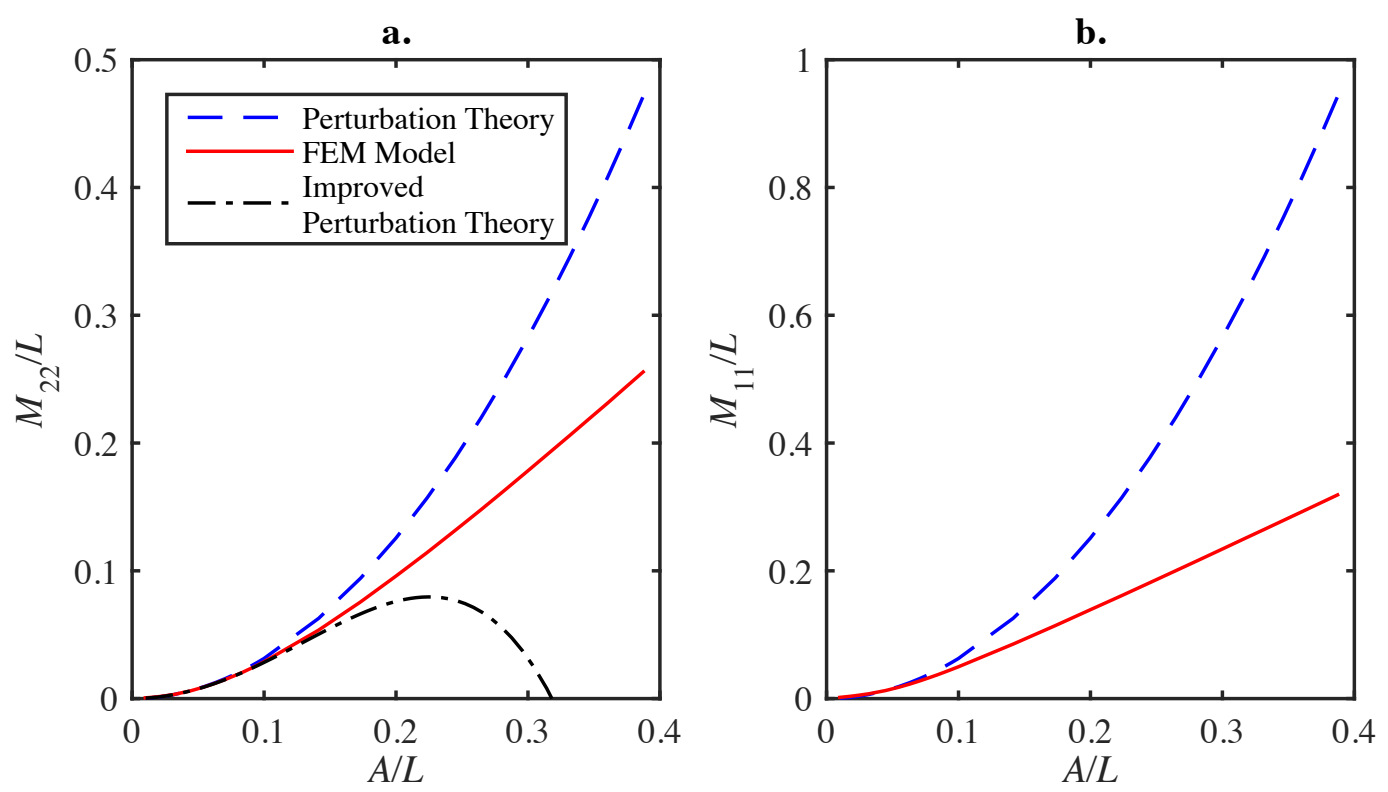

FIG. 5. Comparison between the values for the slip length in the (a) $\hat{z}$ and (b) $\hat{x}$ directions from FEM modeling and the predictions of expression (24). Note the good agreement between the solutions for low aspect ratios. A significant divergence, characterised by a difference between the solutions of more than $10 \%$, occurs for aspect ratios above 0.1 in the $\hat{z}$ direction and 0.56 in the $\hat{x}$ direction. Inclusion of the next order term for flow in the $\hat{z}$ direction in the perturbation expansion delays the divergence to aspect ratios of 0.15 .

significantly for aspect ratios less than 0.056. This case of fluid flow across the grooves of a sinusoidal surface was also considered by Scholle ${ }^{12}$ who obtained a semi-analytic expression for the slip length using complex analysis. The $M_{11}$ values from the FEM model for the sinusoidal surface were compared to the results found by Scholle ${ }^{12}$ (valid for arbitrary aspect ratios), with no difference found for all aspect ratios.

The perturbation theory, expression (22), is only the leading order term of an infinite series. To explore the relative importance of the higher order terms, we follow the procedure of ref. 14 for the sinusoidal surface given by equation (25) to find the next order term for $\tilde{M}_{22}$. Including this extra term, the equation for $\tilde{M}_{22}$ becomes

$$
\tilde{M}_{22}=\frac{A^{2} \pi}{L}-\frac{A^{4} \pi^{3}}{L^{3}} .
$$

Note that, over some limited range of aspect ratios, this does indeed improve the agreement between the perturbation theory and the numerical results (FIG. 5a), but that it also leads 
to a slip length that can become negative, which is unphysical. Only the inclusion of further terms in the series can remedy this. As the development of the perturbation series beyond expression (22) becomes rapidly more cumbersome as further terms are included this means that it is difficult to improve the accuracy of this perturbation method, leaving us without an obvious way to proceed when looking for an analytic calculation of the slip length for surfaces with large aspect ratios.

\section{CONCLUSIONS}

In this work we have found a very simple exact solution for Stokes flow parallel to the grooves of a family of one-dimensionally structured surfaces generated from a conformal map. From these solutions we derived an exact expression for the slip length of the surface and compared these exact values to those computed from a perturbation theory, finding that, despite its approximate nature, the perturbation theory gives results in agreement with the exact expression for the full range of the conformal map. This family of surfaces could be used as the basis to create further surfaces using a linear superposition. Whilst finding the flow above these surfaces should be trivial finding a value for the slip length will be more involved. Likewise the method of using a conformal map can be extended to other conformal maps as long as they are periodic and tend to flat lines as $y$ tends to $\infty$.

We also found good agreement between numerical modelling and the perturbation approximation at low aspect ratios for sinusoidally varying surfaces. In addition we find that the perturbation theory fares less well when the flow is across the structuring, rather than parallel to it, with significant divergence occurring at roughly half the aspect ratio for a similar divergence to the flow along the grooves case.

The form of the mobility tensor initially used (expression (22)) is the first term in an expansion around the case of flow over a flat surface. We constructed the next order term for the simple case of a sinusoidal surface shape, but found that although this improved the results within some small range of aspect ratios, it led at higher aspect ratios to some unphysical values for the slip length that could only be remedied through including higher 
order terms which are in general difficult to compute. Overall this suggests that another method is needed for analytically computing the slip over very wavy surfaces, even in the Stokes regime. For flow across the grooves of one dimensionally structured surfaces this is provided by Scholle ${ }^{12}$ although another technique is required for more general surface structures that vary in both directions.

\section{ACKNOWLEDGMENTS}

We acknowledge financial support from DSTL, and from the Engineering and Physical Sciences Research Council (EPSRC) of the United Kingdom, via the EPSRC Centre for Doctoral Training in Electromagnetic Metamaterials (Grant No. EP/L015331/1).

\section{REFERENCES}

${ }^{1}$ J. P. Rothstein. Slip on Superhydrophobic Surfaces. Annual Review of Fluid Mechanics, 42:89-109, dec 2009.

${ }^{2}$ M. Z. Bazant and O. I. Vinogradova. Tensorial hydrodynamic slip. Journal of Fluid Mechanics, 613:125-134, oct 2008.

${ }^{3}$ S. Lyu, D. C. Nguyen, D. Kim, W. Hwang, and B. Yoon. Experimental drag reduction study of super-hydrophobic surface with dual-scale structures. Applied Surface Science, 286:206-211, dec 2013.

${ }^{4}$ S. Türk, G. Daschiel, A. Stroh, Y. Hasegawa, and B. Frohnapfel. Turbulent flow over superhydrophobic surfaces with streamwise grooves. Journal of Fluid Mechanics, 747:186217, apr 2014.

${ }^{5}$ Y. L. Zhang, H. Xia, E. Kim, and H. B. Sun. Recent developments in superhydrophobic surfaces with unique structural and functional properties. Soft Matter, 8(44):11217, oct 2012.

${ }^{6}$ S. Zhang, X. Ouyang, J. Li, S. Gao, S. Han, L. Liu, and H. Wei. Underwater drag-reducing effect of superhydrophobic submarine model. Langmuir, 31(1):587-93, jan 2015.

${ }^{7}$ D. W. Bechert, M. Bruse, and W. Hage. Experiments with three-dimensional riblets as an idealized model of shark skin. Experiments in Fluids, 28(5):403-412, may 2000.

${ }^{8}$ M. Sasamori, H. Mamori, K. Iwamoto, and A. Murata. Experimental study on drag- 
reduction effect due to sinusoidal riblets in turbulent channel flow. Experiments in Fluids, 55(10):1828, oct 2014.

${ }^{9}$ A. Shapiro. Shape and Flow. Heinemann, 1964.

${ }^{10}$ C. Y. Wang. Drag due to a striated boundary in slow Couette flow. Physics of Fluids, 21(4):697-698, aug 1978.

${ }^{11}$ C. Y. Wang. Stokes flow through a channel with three-dimensional bumpy walls. Physics of Fluids, 16(6):2136, may 2004.

${ }^{12}$ M. Scholle. Creeping couette flow over an undulated plate. Archive of Applied Mechanics (Ingenieur Archiv), 73(11-12):823-840, jun 2004.

${ }^{13}$ A. Niavarani and N. V. Priezjev. The effective slip length and vortex formation in laminar flow over a rough surface. Physics of Fluids, 21(5):052105, may 2009.

${ }^{14}$ K. Kamrin, M. Z. Bazant, and H. A. Stone. Effective slip boundary conditions for arbitrary periodic surfaces: the surface mobility tensor. Journal of Fluid Mechanics, 658:409-437, jul 2010.

${ }^{15}$ P. Dennery and A. Krzywicki. Mathematics for Physicists. Dover Publications Inc., 1996.

${ }^{16}$ NIST Digital Library of Mathematical Functions - Section 4.13 Lambert W-Function . http://dlmf.nist.gov/4.13, Release 1.0.10 of 2015-08-07.

${ }^{17}$ E. Jones, E. Oliphant, and P. Peterson. SciPy: Open Source Scientific Tools for Pythonhttp://www.scipy.org/. 2001.

${ }^{18}$ COMSOL Multiphysics. Comsol Multiphysics User Guide Version 5.1, April 2015. 